\title{
Coexistence of Solitary Fibrous Tumor in the Small Bowel Wall with Mesentery Neuroendocrine Tumor: A First Case Report
}

This article was published in the following Dove Press journal: Pathology and Laboratory Medicine International

\section{Cristo G Salazar ${ }^{\prime}$ Virian D Serei $\mathbb{D}^{\prime}$ Miral S Grandhi ${ }^{2}$ Zhongren Zhou'}

'Department of Pathology \& Laboratory Medicine, Robert Wood Johnson Medical School, Rutgers University, New Brunswick, NJ, 08903, USA; ${ }^{2}$ Division of Surgical Oncology, Rutgers Cancer Institute of New Jersey and Rutgers Robert Wood Johnson Medical School, New Brunswick, NJ, 0890I, USA
Correspondence: Zhongren Zhou Chief of Gastrointestinal Pathology, Department of Pathology \& Laboratory Medicine, Robert Wood Johnson Medical School, 125 Paterson Street,(MEB 233),

New Brunswick, NJ, 08903, USA

Tel + I 732-667-0497

Fax + I 732-235-8I24

Email zz442@rwjms.rutgers.edu
Background: Solitary fibrous tumor (SFT) of the small bowel wall is a rare occurrence with only one case reported to date. SFT in the small bowel wall adjacent to mesenteric neuroendocrine tumor has not been reported, to the best of our knowledge, in the English literature.

Case Presentation: The patient is an 82-year old male with a right perinephric mass incidentally diagnosed during a bladder ultrasound for working-up of chronic urinary tract infections. A follow-up CT of the abdomen and pelvis demonstrated a mass located to the right of the pancreatic head and anterior to the duodenum. A subsequent endoscopic ultrasound (EUS) with fine needle biopsy of the mass diagnosed a low-grade neuroendocrine tumor, which was supported by positivity for CD56, synaptophysin, and chromogranin. An exploratory laparotomy was performed, and the mesenteric mass was identified near the root of the middle colic vessels and laying on top of the duodenum and pancreatic head. A right hemicolectomy with terminal ileum resection was performed en bloc with resection of the mesenteric mass, presumed to be a large lymph node metastasis. A small white-tan, firm nodule was located within the small bowel submucosa and identified, measuring $0.6 \times 0.4$ x $0.3 \mathrm{~cm}$. The mesenteric mass measured $5.5 \times 3.5 \times 3.3 \mathrm{~cm}$ and was in the mesenteric drainage distribution of the small bowel. The mesentery tumor cells were positive for synaptophysin and chromogranin, which supported the diagnosis of neuroendocrine tumor. The small submucosal nodule cells were positive for STAT6, CD34 and CD99, and focally positive for BCL-2, which confirmed the diagnosis of SFT of the small bowel.

Conclusion: We report the first case of SFT within the small bowel submucosa coexisting with a large neuroendocrine tumor within the mesentery.

Keywords: solitary fibrous tumor, neuroendocrine tumor, small bowel, coexistence

\section{Introduction}

Solitary fibrous tumor (SFT) is a rare spindle cell tumor which was first described in 1931 by Klemperer and Robin in the pleura. ${ }^{1-3}$ Klemperer and Rabin described the histologic features of SFT and assigned the name as hemangiopericytoma. More recently the discovery of the SFT NAB2-STAT6 gene fusion ${ }^{4}$ has led to the abandonment of the term hemangiopericytoma in favor of SFT. SFTs are found in almost any site of the body with intra-thoracic, followed by intra-abdominal ${ }^{5,6}$ being the most common locations. The majority of thoracic SFTs are present as asymptomatic masses discovered incidentally by chest imaging. ${ }^{1}$ SFT in the intestinal wall of the small intestine was reported in one case in $2013 .^{7}$ Our case is the second SFT reported to 
arise from the small bowel submucosa and also is the first report of SFT coexisting with a large neuroendocrine tumor in the small bowel mesentery.

\section{Case Report}

The patient is an 82-year old male with a medical history significant for hypertension, morbid obesity, and benign prostatic hyperplasia. For several months he had been suffering from recurrent urinary tract infections, which were initially treated with antibiotics. He underwent an ultrasound of his bladder and kidney for further workup of his chronic urinary tract infections, which coincidently revealed a right perinephric mass. Follow-up abdomen and pelvis CTs demonstrated that the mass measuring $3.8 \times 4.4 \times 4.3 \mathrm{~cm}$ was located to the right of the pancreatic head and anterior to the duodenum (Figure 1A). A subsequent EUS and fine needle biopsy of the mass revealed a low-grade neuroendocrine tumor, which was supported by positivity for CD56, synaptophysin and chromogranin. The Ki67 proliferation rate was $<1 \%$. Based on the imaging findings, this mass likely represented a lymph node near the base of the mesentery caused by lymphatic spread from a separate primary lesion.

The Ga-68 PET/CT full-body scan can capture neuroendocrine tumors (NETs) that overexpress somatostatin receptors and show where the tumors are in the body. Subsequent Gallium 68 Dotatate Scan for our patient demonstrated the large mesenteric nodal lesion to have high uptake 94 (SUV of 94) with no evidence of the location of the primary lesion, additional nodal disease, or distant metastatic somatostatin avid disease. Colonoscopy findings were only significant for diverticulosis and two small benign polyps in the cecum and the ascending colon. Capsule endoscopy also failed to localize a primary lesion, showing only a questionable area of localized inflammation and wall thickening. All preoperative attempts previous mentioned were unsuccessful to find the primary tumor site. Hence, we consented the patient for the operating room with plans for exploratory laparotomy, likely right hemicolectomy with small bowel resection en bloc with mesenteric lymph node resection, and further indicated procedures. The surgery was performed to in order localize and resect the primary lesion en bloc with the mesenteric mass, which had been presumed to be a metastatic lymph node. During the procedure the mesenteric nodule mass was identified. A right hemicolectomy was performed en bloc with resection of the mesenteric mass, which was adherent to the head of the pancreas and duodenum. A small nodule in the terminal ileum was also identified. It was believed to be the primary small bowel
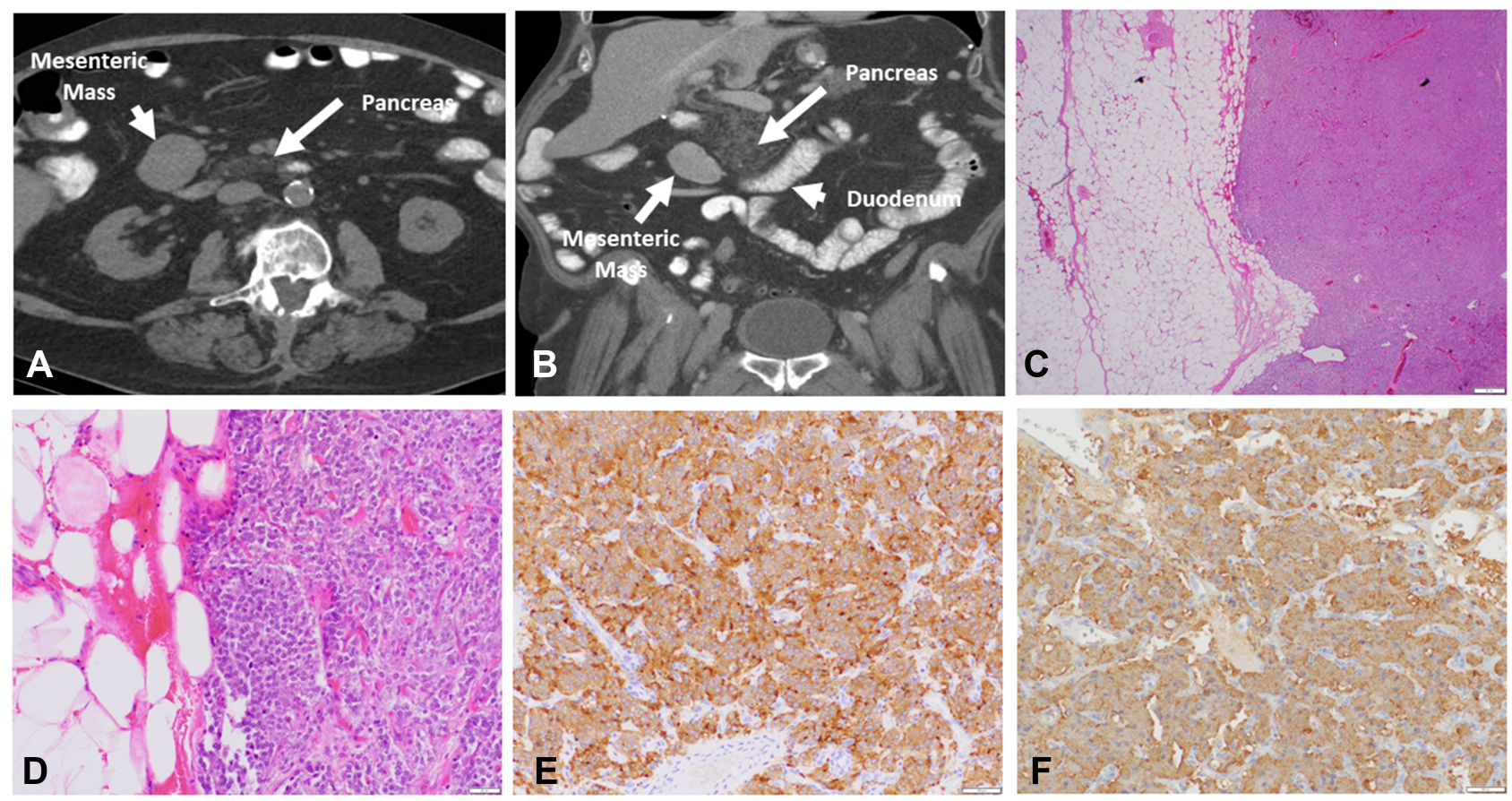

Figure I Metastatic neuroendocrine tumor metastasis in the mesentery. (A, B) CT scan images of abdomen mass close to the right of the pancreatic head and anterior to the duodenum, (A) axial images of the mesenteric mass in relation to the pancreatic head; (B) coronal CT scan images of mesenteric mass in relation to the pancreas and duodenum. (C, D) Neuroendocrine cells in nest with lower nuclear/cytoplasm ratio, bland nuclei with "salt and paper" chromatin in mesentery. (C) 20x and (D) I00x; (E). Neuroendocrine tumor with positive synaptophysin immunostain; (F) neuroendocrine tumor with positive chromogranin. 
neuroendocrine tumor. However, a frozen section of the small bowel lesion was sent in order to confirm it to be a neuroendocrine tumor. The diagnosis was returned as a spindle cell neoplasm.

No other lesion was identified during surgery. The specimen was sent to pathology for diagnosis. The specimen revealed a small white-tan, firm, submucosal nodule measuring $0.6 \times 0.4 \times 0.3 \mathrm{~cm}$. The adjacent mesenteric mass measured $5.5 \times 3.5 \times 3.3 \mathrm{~cm}$ and was attached to the mesentery. The cut surface of the mass was variegated and pink-tan. Further dissection of the mesentery revealed numerous enlarged lymph nodes. The small and large bowel mucosa were unremarkable, demonstrating pink-tan glistening folds.

Microscopically, the smaller nodular lesion in the small bowel submucosa demonstrated bland, spindled fibroblastic cells with small, uniform nuclei arranged in a "patternless" pattern (Figure 2A and B). Small vessels lined with bland, flattened endothelial cells were present within the lesion (Figure 2B). Immunohistochemical stainings showed positive for STAT6, CD34 and CD99, and focally positive for BCL-2, but negative for DOG1, CD117, smooth muscle actin (SMA), and S-100 (Figure 2C-F). Together, the morphology and immunohistochemical stains supported the diagnosis of a submucosal SFT of the small bowel. The diagnosis of a neuroendocrine tumor was also confirmed in the mesenteric mass, consistent with a metastatic neuroendocrine tumor. Morphologically, the tumor consisted of small, round, relatively monomorphic nested cells with "salt and pepper" nuclear chromatin (Figure 1C and D). The cells were positive for synaptophysin and chromogranin immunohistochemical staining with a $\mathrm{Ki67}$ index of less than $2 \%$ (Figure $1 \mathrm{E}$ and F). Thirty additional mesenteric lymph nodes were negative for both neuroendocrine tumor and SFT.

The patient's post-operative course was complicated by bilateral lower extremity edema for which the etiology was unclear. Otherwise, the patient did not experience other gastrointestinal symptoms. The patient was scheduled for surveillance every 6 months for 2-3 years, followed by yearly surveillance for 5 years. This patient provided the written informed consent for the case details to be published. The hospital does not have a limitation for this case report publication.

\section{Discussion}

SFT is a rare mesenchymal tumor first described and named "hemangiopericytoma" in the pleura in $1931 .^{3}$ SFTs are relatively common in the pleura, especially in the visceral leaflet of the pleura. ${ }^{1,8}$ They represent less than $5 \%$ of all pleural tumors. Most SFTs occur in adults,
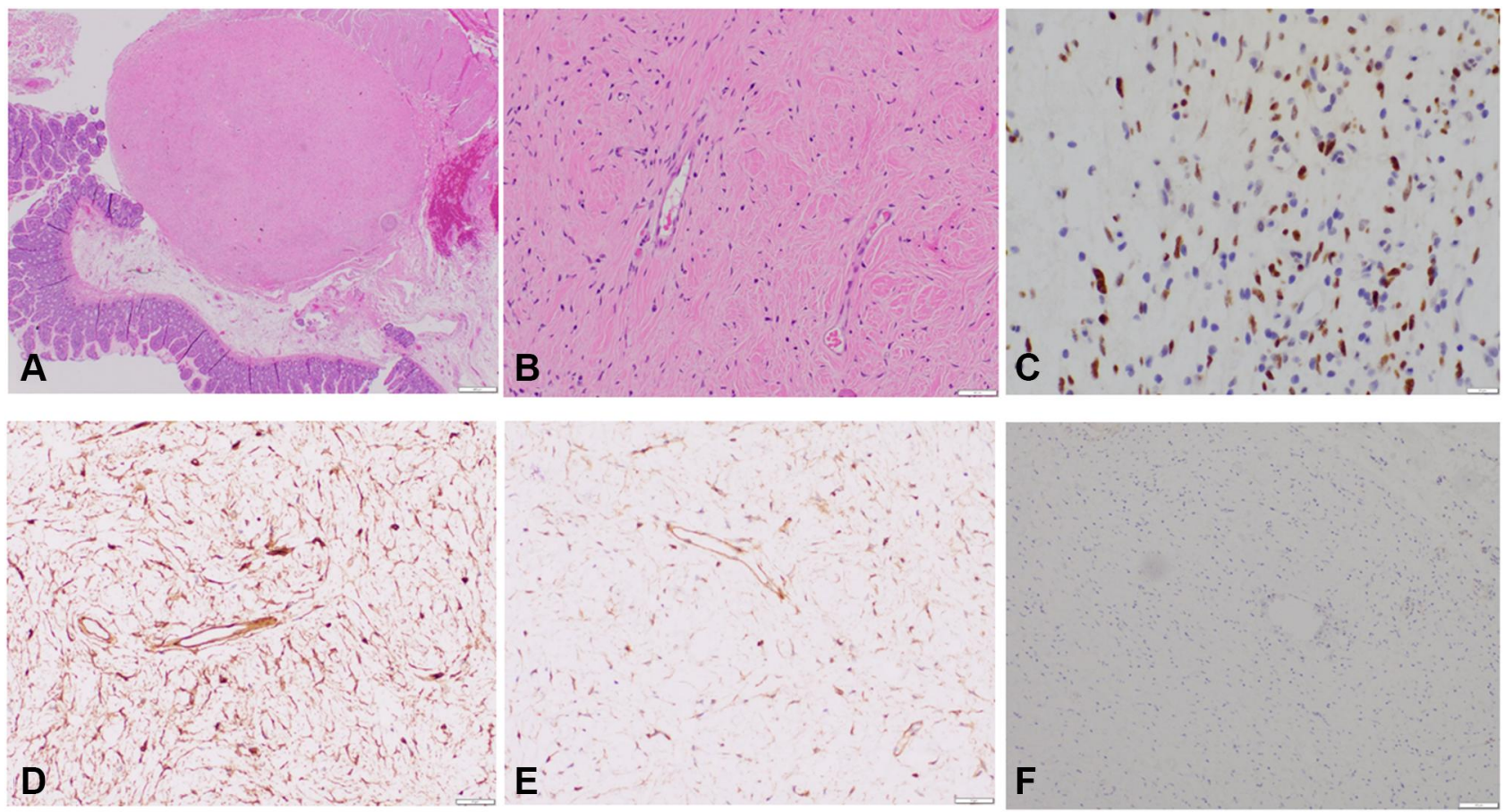

Figure 2 Solitary fibrous tumor (SFT) in small bowel submucosa. (A) In Low power (20x), SFT located within small bowel submucosa; (B) in high power (I00x), tumor cells are spindle cells with elongated small vessels; (C-E) tumor cells are positive for STAT6 (C), CD34 (D), and CD99 (E); (F) tumor cells are negative for DOGI (F). 
with a median age of $45-50$ years. SFTs are now discovered throughout the body, including soft tissue and viscera, albeit with a peculiar predilection for body cavities, including pleura, peritoneum, and meninges. Soft-tissue SFTs represent only $1-2 \%$ of all soft-tissue tumors. ${ }^{1,9}$

Recently, eleven cases of SFTs in the small bowel mesentery have been reported in the literature. ${ }^{2,10-14}$ However, the presence of SFT within the wall of the small bowel has been reported only on one occasion: in 2013 by Yong-Qiao Liu and Jun-Qiu Yue. ${ }^{7}$ They reported the tumor to be incidentally noted in a 26 year old female during cesarean section. The tumor was removed by complete resection and was a well-encapsulated, gray, firm mass of size $3.5 \times 3.0 \times 3.0 \mathrm{~cm}$, located in the muscularis propria of the ileum. The tumor cells were positive for CD34, CD99, vimentin, and BCL-2; focally positive for p53, but uniformly negative for CD117, S-100 protein, and smooth muscle actin (SMA), which confirmed the diagnosis of SFT. Our case is the second SFT identified within the wall of the small bowel. We discovered a small nodule within the small bowel submucosa for a segment of bowel resection of a large mesenteric mass consistent with a neuroendocrine tumor. Upon exploration, no primary lesion corresponding with the metastatic mass was found. Initially, we considered the nodule to be a diminutive gastrointestinal stromal tumor (GIST) with some differential diagnosis of leiomyoma and Schwannoma. However, the spindle tumor cells were negative for the CD117, DOG1, smooth muscle actin and s-100, but positive for CD34. Subsequently, the smaller SFT was thought to be a new diagnosis. We added STAT6, CD99, and BCL-2 immunostain to the panel; the tumor cells showed positive for all three immunostains, which confirmed the diagnosis of SFT. This is the first reported case in which SFT in the small bowel mucosa coexisted with a large neuroendocrine tumor metastasis within the mesentery in the English literature. Since the nodule was so small, the entire nodule was totally submitted without a gross picture. However, this SFT is the smallest SFT reported in the abdomen.

In SFT, there is no clear connection between the tumor's morphology and prognosis, given that SFT behavior is unpredictable. ${ }^{1,8}$ Twenty percent of SFTs possess a malignant variant characterized by larger tumors (more than $5 \mathrm{~cm}$ in diameter), hypercellular and invasive features, nuclear pleomorphism, tissue necrosis, and a high mitotic index greater than 4 mitoses per $10 \mathrm{HPF}$. All SFTs have the potential to become malignant; thus, a systematic mitosis count is recommended. ${ }^{8}$ In our case, the SFT was present with benign characteristics such as smaller size (a diameter of $0.6 \mathrm{~cm}$ ), no associated necrotic zones, no pleomorphism, and a mitotic index of less than $1 \%$ per 10 HPF. A complete excision is the recommended treatment in this case.

Small bowel neuroendocrine tumors (SBNETs) have been increasing in frequency over the past decades and are now the most common type of small bowel tumor. ${ }^{15-17}$ Neuroendocrine tumors have been very well documented, and the coexistence with other tumors, such as gastrointestinal stroma tumor, have been also reported. ${ }^{15}$ The neuroendocrine neoplasms (NEN) can occur in any epithelial organs of the body and are not an infrequent in the gastrointestinal tract (GIT). The presence of NENs in GIT associated with other secondary primary malignancies (SPM) has been considered an exotic event. ${ }^{19}$ One of the theories to explain the association of NEN of gastrointestinal tract and secondary primary synchronous in GIT is the ability of NENs to generate synchronous tumors by autocrine and paracrine effect. Autocrine and paracrine signaling leading to stimulation of tumor cell growth is a common theme in human cancers. ${ }^{20}$

The uniqueness of this case is the coexistence of neuroendocrine tumors with SFT, which had not been reported yet in the English literature. Only one pelvic malignant SFT was reported to metastasize to the liver with transdedifferentiated to a high-grade neuroendocrine carcinoma. ${ }^{18}$ Therefore, our case is the first case to report the coexistence of SFT in terminal ileum and mesentery neuroendocrine tumors. However, it is important to mention that there was no connection identified between the two tumors.

\section{Disclosure}

The authors report no conflicts of interest in this work.

\section{References}

1. Davanzo B, Emerson RE, Lisy M, Koniaris LG, Kays JK. Solitary fibrous tumor. Transl Gastroenterol Hepatol. 2018;3:94. doi:10.21037/ $\operatorname{tgh} .2018 .11 .02$

2. Lau MI, Foo FJ, Sissons MC, Kiruparan P. Solitary fibrous tumor of small bowel mesentery: a case report and review of the literature. Tumori. 2010;96(6):1035-1039. doi:10.1177/548.6529

3. Klemperer P. Primary neoplasms of the pleura: a report of five cases. Arch Pathol. 1931;11:385-412.

4. Robinson DR, Wu YM, Kalyana-Sundaram S, et al. Identification of recurrent NAB2-STAT6 gene fusions in solitary fibrous tumor by integrative sequencing. Nat Genet. 2013;45(2):180-185. doi:10.10 38/ng.2509

5. Hasegawa T, Matsuno Y, Shimoda T, Hasegawa F, Sano T, Hirohashi S. Extrathoracic solitary fibrous tumors: their histological variability and potentially aggressive behavior. Hum Pathol. 1999;30 (12):1464-1473. doi:10.1016/S0046-8177(99)90169-7 
6. Gold JS, Antonescu CR, Hajdu C, et al. Clinicopathologic correlates of solitary fibrous tumors. Cancer. 2002;94(4):1057-1068. doi: $10.1002 /$ cncr. 10328

7. Liu YQ, Yue JQ. Intramural solitary fibrous tumor of the ileum: a case report and review of the literature. J Cancer Res Ther. 2013;9(4):724-726. doi:10.4103/0973-1482.126469

8. Schirosi L, Lantuejoul S, Cavazza A, et al. Pleuro-pulmonary solitary fibrous tumors: a clinicopathologic, immunohistochemical, and molecular study of 88 cases confirming the prognostic value of de perrot staging system and p53 expression, and evaluating the role of c-kit, BRAF, PDGFRs (alpha/beta), c-met, and EGFR. Am J Surg Pathol. 2008;32(11):1627-1642. doi:10.1097/PAS.0b013e31817a8a89

9. Goodlad JR, Fletcher CD. Solitary fibrous tumour arising at unusual sites: analysis of a series. Histopathology. 1991;19(6):515-522. doi:10.1111/j.1365-2559.1991.tb01499.x

10. Cantarella F, Graziosi L, Cavazzoni E, Donini A. Small bowel mesentery solitary fibrous tumor. A rare neoplasia in a young male. G Chir. 2012;33(8-9):271-273.

11. Chiroque L, Arenas J, Luyo-Rivas A. Solitary fibrous tumor confined to the small bowel (ileum) mesentery. Rev Gastroenterol Mex. 2013;78(4):259-260. doi:10.1016/j.rgmx.2013.04.002

12. Zhang GJ, Li RT, Zhou Y, et al. Solitary fibrous tumor of small bowel mesentery with postoperative bowel obstruction: a case report and review of literature. Int J Clin Exp Pathol. 2015;8(9):11691-11697.

13. Nishida K, Ubukata H, Konishi S, et al. A giant solitary fibrous tumor of the mesentery: a case report and literature review. World J Surg Oncol. 2015;13:17. doi:10.1186/s12957-014-0422-4
14. Lucas CE, Ledgerwood AM. Malignant solitary fibrous tumor of the intestine with refractory hypoglycemia (doege potter syndrome). $J \mathrm{Am}$ Coll Surg. 2006;203(3):398. doi:10.1016/j.jamcollsurg.2006.01.021

15. Howe JR, Cardona K, Fraker DL, et al. The surgical management of small bowel neuroendocrine tumors: consensus guidelines of the North American neuroendocrine tumor society. Pancreas. 2017;46 (6):715-731. doi:10.1097/MPA.0000000000000846

16. Oronsky B, Ma PC, Morgensztern D, Carter CA. Nothing but NET: a review of neuroendocrine tumors and carcinomas. Neoplasia. 2017;19(12):991-1002. doi:10.1016/j.neo.2017.09.002

17. Martini C, Sturniolo GC, De Carlo E, et al. Neuroendocrine tumor of small bowel. Gastrointest Endosc. 2004;60(3):431. doi:10.1016/ S0016-5107(04)01702-X

18. Lu C, Alex D, Benayed R, Rosenblum M, Hameed M. Solitary fibrous tumor with neuroendocrine and squamous dedifferentiation: a potential diagnostic pitfall. Hum Pathol. 2018;77:175-180. doi:10.1016/j.humpath.2017.12.024

19. Parra-Medina R, Moreno-Lucero P, Jimenez-Moreno J, ParraMorales AM, RomeroRojas A. Neuroendocrine neoplasms of gastrointestinal tract and secondary primary synchronous tumors: a systematic review of case reports. Casualty or causality? PLoS One. 2019;14(5):e0216647. doi:10.1371/journal.pone.0216647

20. Prommegger R, Ensinger C, Steiner P, Sauper T, Profanter C, Margreiter R. Neuroendocrine tumors and second primary malignancy--a relationship with clinical impact? Anticancer Res. 2004;24(2C):1049-1051.

\section{Publish your work in this journal}

Pathology and Laboratory Medicine International is a peer-reviewed, open access journal focusing on innovative basic research and translational research related to pathology or human disease. The journal includes original research, updates, case reports, reviews and commentaries on current controversies. The manuscript management system is completely online and includes a very quick and fair peer-review system. Visit http://www.dovepress.com/testimonials.php to read real quotes from published authors. 